\title{
Dynamical Modeling using Evolutionary Algorithms
}

\author{
Anne Mathieu \\ Instituto de Astrofisica de Andalucia, Calle Camino Bajo de Huetor 24, \\ Granada 18008, Spain
}

\begin{abstract}
I will present a new method of dynamical modeling of stellar systems based on evolutionary algorithms. The technique will be illustrated with an application to the problem of recovering the gravitational potential of a thin galactic disc from kinematic observables in a non-parametric way.
\end{abstract}

\section{Introduction}

All the intrinsic and observable properties of stellar systems are determined by the phase space distribution function and the gravitational potential of these systems. Thus the quest for inferring the dynamics of galaxies from a set of observables is an essential problem of dynamical astronomy. In this framework a fundamental task is to develop algorithms for dynamical modeling that estimate distribution functions and gravitational potentials of galaxies consistent with their observed properties. In this paper we present a method of dynamical modeling based on an evolutionary algorithm that can be used for estimating the gravitational potential of a thin axisymmetric disc galaxy from kinematic observables.

\section{Dynamics of a thin axisymmetric disc galaxy}

For an infinitely thin axisymmetric disc the phase space distribution function is a function of the two integrals of motion, the energy and the angular momentum around the symmetry axis of the disc, and the axisymmetric gravitational potential is a function of radius. For such systems, the observable containing the most information is the line-of-sight velocity distribution (hereafter LOSVD). It is a function of the projected radius and the line-of-sight velocity which can be routinely derived from spectroscopic observations of disc galaxies. If the disc is inclined, there is a simple relation linking the line-of-sight velocity distribution to the phase space distribution function: it is a one-dimensional Abel transform for which the inversion formula is known analytically. Therefore if one can measure the line-of-sight velocity distribution of a thin disc, one can derive theoretically the distribution function of the disc assuming the gravitational potential is known a priori.

One can show that the distribution function is completely specified by both the part of the LOSVD that is above the rotation curve and by the part below the rotation curve (see Mathieu \& Merrifield 2000; Merrifield \& Kuijken 1994). 
This means that the two parts of the LOSVD are symmetrically related and the dividing line between the two regions is the rotation curve (scaled to a factor depending on the inclination angle). Thus one can use this symmetry to uniquely infer the rotation curve as a function of radius and then derive the potential using the estimate for the inferred rotation curve. We develop a method that searches for the best fit rotation curve using an evolutionary algorithm.

\section{Inference of the gravitational potential of a thin inclined disc}

The algorithm aims to find the rotation curve that minimizes the difference of the LOSVD values for pairs of corresponding points in the parts of the LOSVD below and above the rotation curve. The parameters to determine are the values of the slope of the rotation curve at discrete radii. As the number of parameters is large, conventional optimization methods fail but evolutionary algorithms are well suited to this kind of non-linear problems. We adopt a self-organizing migrating algorithm. The algorithm starts with a population of $\mathrm{N}$ possible models of physical rotation curves created randomly. For each rotation curve, the algorithm calculates the sum of the squared differences between the parts of the LOSVD above and below the rotation curve. The smaller this sum (called fitness) is, the better the estimate of the rotation curve is. Then the algorithm selects a random subspace of the space of the parameters and makes each model travel towards the best rotation curve found so far by discrete steps (this process is called a migration loop) in this subspace. The fitness of each new model is evaluated and the model with the smallest fitness becomes the new best model. Then the whole process of migration loop and determination of best model is repeated again until the fitness is small enough.

To test the method, we consider a toy model of a thin axisymmetric disc with a two-integral distribution function like the one described in Kuijken \& Tremaine (1991). We adopt a softened isothermal sphere as the true gravitational potential of the disc. We calculate the LOSVD assuming an arbitrary inclination of the disc and try to recover the correct form of the rotation curve from the LOSVD only. The evolutionary algorithm succeeds in approaching the true rotation curve closely with a small number of iterations (Mathieu 2003).

Acknowledgments. This research has been supported by a Marie Curie Fellowship of the European Community programme 'Improving Human Research Potential and the Socio-economic Knowledge Base' under contract number HPMF-CT-2000-00911.

\section{References}

Kuijken, K., \& Tremaine, S. 1991, in Dynamics of Disc Galaxies, Varberg Castle Sweden (Knudsen), 71

Mathieu, A. 2003, in preparation

Mathieu, A., Merrifield, M.R. 2000, MNRAS, 318, 40

Merrifield, M.R., Kuijken, K. 1994, ApJ, 432, 575 